\title{
Approximate Stationary Density of the Nonlinear Dynamical Systems Excited with White Noise
}

\author{
Serkan Günel \\ Dokuz Eylül University \\ Dept. of Electrical and Electronics Engineering \\ İzmir, Turkey \\ Email: serkan.gunel@eee.deu.edu.tr
}

\author{
F. Acar Savac1 \\ İzmir Institute of Technology \\ Dept. of Electrical and Electronics Engineering \\ İzmir, Turkey \\ Email: acarsavaci@iyte.edu.tr
}

\begin{abstract}
In this paper, obtaining approximate solution of Fokker-Planck-Kolmogorov (FPK) Equation using compactly supported functions has been discussed. With specific choice of such functions as piecewise multivariable polynomials which are supported on ellipsoidal regions, the parameters to be determined can be considerably decreased compared to MultiGaussian Closure scheme [1]. An example commonly considered in the literature has been analyzed and the proposed method has been compared with the Multi-Gaussian Closure scheme. The simulation results indicate that the new scheme is quite successful even if the driving noise is not white Gaussian, but has an exponential correlation function with small correlation time.
\end{abstract}

\section{INTRODUCTION}

The effect of indeterministic inputs on dynamical systems can be considered in the view of Fokker-Planck-Kolmogorov (FPK) formalism [2], [3]. This formalism gives one the ability to transform the random dynamical systems evolving in the state space under the influence of Gaussian White noise either in additive or in multiplicative form, to a new dynamical system evolving in the space of probability density functions stated in terms of a linear parabolic partial differential equation, the Fokker-Planck-Kolmogorov equation. It is known that an exact solution of the FPK equation is generally hard to find and in some special cases exact solution is known [4][9]. Many approximate methods have been developed, such as the variational methods based on the eigenfunction expansion of the probability density function (pdf) [10], [11], iterative methods based on the solution of an integral equation [2], [12], the maximum entropy approach originated by the classical work of Janes [13] in which the solution of the FPK equation is presented by an infinite dimensional dynamical system of the moments of the pdfs [14], [15] and weighted residual schemes [16]-[20]. A simple approach is the Gaussian Closure (GC) method where the solution is assumed to be Gaussian and the parameters of the Gaussian pdf are chosen in order to minimize the approximation error. This approach has been extended to Multi-Gaussian case in which the solution is assumed to be the sum of Gaussian pdfs [1]. Another variant of this method called Exponential Closure is to use the exponential functions of polynomials of the state variables, instead of Gaussian assumption [17], [18]. In these methods, solution of the FPK equation in the weak sense is reduced to a solution of nonlinear algebraic equations.

The Gaussian Closure is unsuitable when the system is highly nonlinear or when multiplicative random excitations exist. In order to eliminate these drawbacks, the Multi-Gaussian Closure method is used, but in this case the number of free parameters to be determined is very much, so that the method may not be feasible for high dimensional systems.

In this paper, the FPK equation arising from the stochastic dynamical system driven with Gaussian white noise has been introduced and a new method based on the weighted residual scheme has been proposed, in which compactly supported multivariable polynomial functions (CSP) have been used to simplify the problem of obtaining and solving nonlinear algebraic equations which represent the FPK equation. The example of bistable system is given to clarify the usefulness of this newly proposed method.

\section{The FoKKer-Planck-Kolmogorov EQuation}

Consider the following stochastic differential equation

$$
\begin{aligned}
\frac{\mathrm{d} \mathbf{x}}{\mathrm{d} t} & =f(\mathbf{x})+g(\mathbf{x}) \eta \\
\mathbf{x}(0) & =\mathbf{x}_{0}
\end{aligned}
$$

where $t \geq 0, \mathbf{x}=\left(x_{1} \cdots x_{n}\right)^{T} \in \mathbb{R}^{n}, f(\mathbf{x}) \in \mathbb{R}^{n}, g(\mathbf{x}) \in \mathbb{R}^{n \times n}$ and $\eta(t)$ is the white noise defined as $\eta(t) \triangleq\left(\begin{array}{lll}\frac{\mathrm{d} \omega_{1}}{\mathrm{~d} t} & \cdots & \frac{\mathrm{d} \omega_{n}}{\mathrm{~d} t}\end{array}\right)^{T}$ where $\omega_{i},(i=1, \ldots, n)$ are 1-D Wiener processes with $E\left\{\omega_{i}(t)\right\}=0, E\left\{\omega_{i}(t) \omega_{i}(t+\tau)\right\}=2 \delta(\tau)$, and $E\{\cdot\}$ denotes the expectation operator.

The density function $p(\mathbf{x}, t) \in \mathrm{D}\left(\mathbb{R}^{\mathrm{n}}\right)$ of the states $\mathbf{x}$, at time $t$ can be defined as

$$
\operatorname{prob}\left\{\mathbf{x}(t) \in B \subset \mathbb{R}^{n}\right\}=\int_{B} p(\mathbf{z}, t) \mathrm{d} \mathbf{z}
$$

where $\operatorname{prob}\{\cdot\}$ denotes the probability, $\mathrm{D}\left(\mathbb{R}^{n}\right) \triangleq$ $\left\{p \in \mathrm{L}^{1}\left(\mathbb{R}^{n}\right) \mid p \geq 0\right.$ and $\left.\|p\|_{\mathrm{L}^{1}}=1\right\}$ denotes the set of density functions and $p(\mathbf{x}, t)$ satisfies

$$
\lim _{\|\mathbf{x}\| \rightarrow \pm \infty} p(\mathbf{x}, t)=0 \quad \forall t \geq 0
$$

Theorem (Fokker-Planck-Kolmogorov [3]): If the functions $g_{i j}, \frac{\partial g_{i j}}{\partial x_{k}}, \frac{\partial^{2} g_{i j}}{\partial x_{k} \partial x_{l}}, f_{i}, \frac{\partial f_{i}}{\partial x_{j}}, \frac{\partial p}{\partial t}, \frac{\partial p}{\partial x_{i}}, \frac{\partial^{2} p}{\partial x_{i} \partial x_{j}}$ are continuous for 
$t>0$ and $\mathbf{x} \in \mathbb{R}^{n}$, and if $f_{i}, g_{i j}$ and their first derivatives are bounded, then $p(\mathbf{x}, t) \in \mathrm{D}\left(\mathbb{R}^{n}\right)$ satisfies

$$
\begin{aligned}
\frac{\partial p}{\partial t} & =\frac{1}{2} \sum_{i, j=1}^{n} \frac{\partial^{2}}{\partial x_{i} \partial x_{j}}\left(a_{i j} p\right) \\
& -\sum_{i=1}^{n} \frac{\partial}{\partial x_{i}}\left(f_{i} p\right), \forall t>0, \mathbf{x} \in \mathbb{R}^{n} \\
p(\mathbf{x}, 0) & =p_{0}(\mathbf{x}), \quad p_{0}(\mathbf{x}) \in \mathrm{D}\left(\mathbb{R}^{n}\right)
\end{aligned}
$$

where $a_{i j}(\mathbf{x}) \triangleq \sum_{k=1}^{n} g_{i k}(\mathbf{x}) g_{j k}(\mathbf{x}) \quad(i, j=1,2, \ldots, n)$, which is always nonnegative, and $p_{0}(\mathbf{x})$ is the initial density of the states.

Defining the FPK operator as

$$
\begin{aligned}
\mathcal{L}_{t} & \triangleq \frac{1}{2} \sum_{i=1}^{n} \sum_{j=1}^{n} \frac{\partial^{2}}{\partial x_{i} \partial x_{j}}\left(a_{i j}(\mathbf{x})(\cdot)\right) \\
& -\sum_{i=1}^{n} \frac{\partial}{\partial x_{i}}\left(f_{i}(\mathbf{x})(\cdot)\right)-\frac{\partial}{\partial t}(\cdot)
\end{aligned}
$$

and the system given in (1) then FPK equation (4) can be written as

$$
\mathcal{L}_{t} p(\mathbf{x}, t)=0 \quad \mathbf{x} \in \mathbb{R}^{n}, t>0 .
$$

The stationary density $p_{\mathrm{st}}(\mathbf{x})$, if exists, is defined by the limit

$$
\lim _{t \rightarrow \infty} p(\mathbf{x}, t) \triangleq p_{\text {st }}(\mathbf{x}) \quad \forall p_{0}(\mathbf{x}) \in \mathrm{D}\left(\mathbb{R}^{n}\right)
$$

In the stationary case, the FPK operator can be defined as

$$
\mathcal{L} \triangleq \frac{1}{2} \sum_{i=1}^{n} \sum_{j=1}^{n} \frac{\partial^{2}}{\partial x_{i} \partial x_{j}}\left(a_{i j}(\mathbf{x})(\cdot)\right)-\sum_{i=1}^{n} \frac{\partial}{\partial x_{i}}\left(f_{i}(\mathbf{x})(\cdot)\right)
$$

and hence, the stationary density should satisfy the FPK equation

$$
\mathcal{L} p_{\mathrm{st}}(\mathbf{x})=0 \quad \forall p_{0}(\mathbf{x}) \in \mathrm{D}\left(\mathbb{R}^{n}\right)
$$

together with the boundary condition (3). Multiplying both sides of (9) with an arbitrary test function $h: \mathbb{R}^{n} \rightarrow \mathbb{R}$ of Hilbert Space $\mathcal{H}$ with the usual inner product defined by $\langle f(\mathbf{x}), g(\mathbf{x})\rangle \triangleq \int_{\mathbb{R}^{n}} f(\mathbf{x}) g(\mathbf{x}) \mathrm{d} \mathbf{x} \quad \forall f, g \in \mathcal{H}$, and integrating both sides over the whole domain yields the variational equation

$$
\left\langle\mathcal{L} p_{\text {st }}(\mathbf{x}), h(\mathbf{x})\right\rangle=0
$$

Clearly, all solutions of (9) are the solutions of the variational equation (10), but the inverse statement, in general, is not true. Finding solution of the infinite dimensional variational problem is almost as demanding as the solution of the original problem. In practice, a finite set of linearly independent test functions, $\mathcal{Y} \triangleq\left\{h_{k} \in \mathcal{H}\right\}_{k=1}^{N}$ are chosen in order to satisfy

$$
\left\langle\mathcal{L} p_{\mathrm{st}}(\mathbf{x}), h_{k}(\mathbf{x})\right\rangle=0, \quad k=1, \ldots, N
$$

The solution $p_{\mathrm{st}}$, satisfying (11) is called the solution of (9) in the weak sense. The error between the exact solution of the variational problem and the weak sense solution vanishes in the space spanned by $\left\{h_{k}\right\}_{k=1}^{N}$.

However, in practice, the stationary states of the stable systems are trapped in a specific compact subspace of $\mathbb{R}^{n}$, which implies that the integration over the whole domain is unnecessary. This fact is the inspiration of the newly proposed method based on the compactly supported pdfs.

\section{A. Approximation of the Solution of the FPK Equation Using Compactly Supported Polynomials}

Assuming that the solution of (9) is approximated by a linear combination of compactly supported functions as

$$
p_{\vartheta}(\mathbf{x})=\sum_{i=1}^{K} \kappa_{i} \varphi_{\vartheta_{i}}(\mathbf{x})
$$

where $\kappa_{i} \geq 0, \forall i, \sum_{i} \kappa_{i}=1$ and the set of parameters $\vartheta \triangleq$ $\left\{\vartheta_{i}, i=1, \cdots, K\right\}$.

$\varphi_{\vartheta_{i}}(\cdot) \mathrm{s}$ are the single bump densities supported in the domain $\Omega_{i}$, i.e.

$$
\begin{aligned}
& \varphi_{\vartheta_{i}}(\mathbf{x}) \neq 0, \forall \mathbf{x} \in \Omega_{i}, \\
& \varphi_{\vartheta_{i}}(\mathbf{x})=0, \forall \mathbf{x} \notin \Omega_{i}, \text { and } \forall \mathbf{x} \in \partial \Omega_{i}
\end{aligned}
$$

where $\partial \Omega_{i}$ denotes the boundary of $\Omega_{i}$. The solution of (9) can be approximately constructed using such compactly supported functions as given in the sequel.

Multiplying with the test functions and integrating yields

$$
\int_{\mathbb{R}^{n}} \mathcal{L} p_{\vartheta}(\mathbf{x}) h_{k}(\mathbf{x}) \mathrm{d} \mathbf{x}=\sum_{i=1}^{K} \kappa_{i} \int_{\Omega_{i}} \mathcal{L} \varphi_{\vartheta_{i}}(\mathbf{x}) h_{k}(\mathbf{x}) \mathrm{d} \mathbf{x}
$$

Using (11), a set of equations,

$$
\begin{aligned}
& \Upsilon_{0} \equiv \sum_{i=1}^{K} \kappa_{i}=1 \\
& \Upsilon_{k} \equiv \sum_{i=1}^{K} \kappa_{i} \Upsilon_{k, i}=0, \quad k=1,2, \ldots,(N-1)
\end{aligned}
$$

is obtained, where $\Upsilon_{k, i} \triangleq \int_{\Omega_{i}} \mathcal{L} \varphi_{\vartheta_{i}}(\mathbf{x}) h_{k}(\mathbf{x}) \mathrm{d} \mathbf{x},(i=1, \ldots, K)$. Solving the system of equations (14) yields the best approximation of the solution where the error between the exact pdf and the approximated pdf vanishes on the space spanned by the test functions $\left\{h_{k}\right\}_{k=1}^{N-1}$.

A family of functions in the form of compactly supported multivariable polynomials (CSP) are given by

$$
\begin{aligned}
& \varphi_{\left\{\mu_{i}, \sigma_{i}\right\}_{i=1}^{n}}(\mathbf{x})= \begin{cases}c \cdot\left(-1+\sum_{i=1}^{n} \frac{\left(x_{i}-\mu_{i}\right)^{2}}{\sigma_{i}^{2}}\right)^{2}, & \mathbf{x} \in \Omega_{i} \\
0 & \text { otherwise }\end{cases} \\
& \Omega_{i} \triangleq\left\{\begin{array}{l|l}
\mathbf{x} \in \mathbb{R}^{n} & \sum_{i=1}^{n} \frac{\left(x_{i}-\mu_{i}\right)^{2}}{\sigma_{i}^{2}} \leq 1
\end{array}\right\}
\end{aligned}
$$

where $\left\{\mu_{i}\right\}_{i=1}^{n}$ and $\left\{\sigma_{i}\right\}_{i=1}^{n}$ are the center and axes lengths of the $n$ dimensional ellipsoids respectively, and the $c$ is the normalization constant chosen such that $\varphi_{\left\{\mu_{i}, \sigma_{i}\right\}}(\mathbf{x}) \mathrm{s}$ are pdfs. With additional $n-1$ parameters, ellipsoidal regions can be rotated around the ellipsoid center which results in extra degrees of freedom. The advantage of using such a simple polynomial form of (II-A) has been demonstrated in Section III. 


\section{EXAMPLE}

Let the system be

$$
\dot{x}=f(x)+\sqrt{\alpha} \eta(t)=\frac{1}{2}\left(x-x^{3}\right)+\sqrt{\alpha} \eta(t)
$$

then the corresponding FPK Operator is given by

$$
\mathcal{L} p=-\frac{1}{2}\left(1-3 x^{2}\right) p(x)-\frac{1}{2}\left(x-x^{3}\right) \frac{d p(x)}{d x}+\frac{\alpha}{2} \frac{d^{2} p(x)}{d x^{2}}
$$

For $\alpha=1$, the exact solution is known to be [9]

$$
p_{\text {ex }}(x)=\frac{2}{\pi\left(I_{-\frac{1}{4}}\left(\frac{1}{16}\right)+I_{\frac{1}{4}}\left(\frac{1}{16}\right)\right)} \mathrm{e}^{-\frac{1}{16}+\frac{1}{4} x^{2}\left(1-\frac{x^{2}}{2}\right)}
$$

where $I_{n}(x)$ is the Bessel Function of the first kind.

The solution for the stationary density in form of the CSP is

$$
\begin{gathered}
p_{\mathrm{CSP}}(x)=\sum_{i=1}^{K} \kappa_{i} \varphi_{\mu_{i}, \sigma_{i}}(x) \\
\varphi_{\mu_{i}, \sigma_{i}}(x)= \begin{cases}\frac{15}{16 \sigma_{i}}\left(\left(\frac{x-\mu_{i}}{\sigma_{i}}\right)^{2}-1\right)^{2}, & \mu_{i}-\sigma_{i} \leq x \leq \mu_{i}+\sigma_{i} \\
0, & \text { otherwise }\end{cases}
\end{gathered}
$$

Nonlinear algebraic equations take the form

$$
\Upsilon_{k, i}(x)=\int_{\mu_{i}-\sigma_{i}}^{\mu_{i}+\sigma_{i}} \mathcal{L} \varphi_{\mu_{i}, \sigma_{i}}(x) h_{k}(x) d x
$$

and choosing $h_{k} \triangleq x^{k}(k=1,2)$, then evaluating (21) yields

$$
\begin{aligned}
& \Upsilon_{1, i}=\frac{\mu_{i}}{2}-\frac{\mu_{i}{ }^{3}}{2}-\frac{3 \mu_{i} \sigma_{i}{ }^{4}}{14} \\
& \Upsilon_{2, i}=\alpha+\mu_{i}{ }^{2}-\mu_{i}{ }^{4}+\frac{\sigma_{i}{ }^{4}}{7}-\frac{6 \mu_{i}{ }^{2} \sigma_{i}{ }^{4}}{7}-\frac{\sigma_{i}{ }^{8}}{21}
\end{aligned}
$$

For the case $K=3, \alpha=1$, solving (14) numerically yields:

$$
\begin{array}{lll}
\kappa_{1} \approx 0.4789 & \kappa_{2} \approx 0.0423 & \kappa_{3} \approx 0.4789 \\
\sigma_{1} \approx 1.3623 & \sigma_{2} \approx 2.8509 & \sigma_{3} \approx 1.3623 \\
\mu_{1} \approx-0.8801 & \mu_{2} \approx 0.0000 & \mu_{3} \approx 0.8801
\end{array}
$$

The comparison of the result, MGC scheme for $K=3$ and simulation data are shown in Figure 1. The simulation is done using a $5^{\text {th }}$ order Dormand-Prince solver [21] with fixed step size of time increments, $\Delta t=1 \times 10^{-3} \mathrm{sec}$, , and $t_{\max }=120 \mathrm{sec}$., and first 20 seconds of the simulation data are ignored to allow the transients to die out. The Gaussian white noise source is replaced by a zero mean bandlimited white noise, $\xi(t)$, with $E\{\xi(t) \xi(t+\tau)\}=\frac{\alpha}{t_{c}} \mathrm{e}^{-\frac{|\tau|}{t_{c}}}$, the correlation time $t_{c}=1 \times 10^{-3} \mathrm{sec}$. The pdf is estimated using the Parzen's estimator [22]:

$$
\begin{gathered}
\hat{p}(x) \triangleq \frac{1}{N h(N)} \sum_{i=1}^{N} g\left(\frac{x-x\left(t_{i}\right)}{h(N)}\right) \\
g(x)=\frac{1}{\sqrt{2 \pi}} \mathrm{e}^{-\frac{x^{2}}{2}}, \quad h(n)=\frac{1}{\sqrt{n}}
\end{gathered}
$$


Remark 1: Although the noise source used in the simulations is not exactly white since hypothetically defined white noise is not realizable physically (which implies that the transition probability of the states is no more Markovian, the FPK equation is not valid and requires generalization [23]), the proposed scheme estimates the pdf of the states in both examples quite successfully. Using compactly supported polynomials as in (II-A) has an advantage that it needs at most $3 K n$ parameters to be determined totally (i.e., $n, n, n-1$ parameters for axis lengths, the center point and the axis rotations of the $n$ dimensional ellipsoids, respectively, together with the weights of the base functions). On the other hand, Multi-Gaussian Closure scheme needs $K \frac{(n+2)(n+1)}{2}$ parameters, in the same resolution. Further reduction in the number of parameters to be determined is possible if the ellipsoid axes are chosen to be equal and the rotations are omitted, which may be suitable in some cases.

Remark 2: It should be noted that, because of the properties of the base functions, the density obtained by the proposed method does not approximate the tail of the exact solution well. Hence the proposed form of approximate densities may not be suitable for reliability analysis. Since, the qualitative behavior of the system under consideration is characterized not only by the tails but the pdf as a whole, the proposed scheme well characterize the qualitative behavior.

The results indicate that the effect of choosing compactly supported multivariable polynomials as base functions is in such way that noise is filtered. In other words, although the analysis are made on the assumption that the source is white, the proposed approximation ensembles the states as if the noise is slightly correlated, which is practically the case, and hence the proposed approximation resembles to Parzen's estimation obtained by the simulation data for a realistic correlation time rather than the exact solution.

\section{Conclusions}

In this paper, the new method of obtaining set of nonlinear algebraic equations whose solution is the parameters of compactly supported approximate solution of FPK equation in the weak sense for the nonlinear systems driven with Gaussian white noise, has been presented. With the specific choice of compactly supported polynomial functions, it has been possible to choose the number of parameters to be determined considerably smaller than the parameters of the MGC and Exponential Closure schemes as the dimensionality of the system increases. The errors between the exact solution and approximate solutions have been compared and it is seen that the pdfs obtained by the proposed method is very close to the exact solution, and is in agreement with the pdf estimated by the Parzen's estimator obtained from the the simulation data, even if the noise source driving the system is not white. The approximate pdfs of the CSP method also confirm the expected physical behavior as the noise power is changed.

\section{REFERENCES}

[1] G.-K. Er, "Multi-Gaussian closure method for randomly excited nonlinear systems," International Journal of Nonlinear Mechanics, vol. 33, no. 2, pp. 201-204, 1998.

[2] H. Risken, The Fokker-Planck Equation: Methods of Solution and Applications, $2^{\text {nd }}$ ed., H. Haken, Ed. Berlin, Germany: Springer-Verlag, 1996.

[3] A. Lasota and M. C. Mackey, Chaos, Fractals and Noise: Stochastic Aspects of Dynamics, $2^{\text {nd }}$ ed., ser. Applied Mathematical Sciences. Berlin, Germany: Springer-Verlag, p. 359, 1994.

[4] R. Wang, K. Yasuda, and Z. Zhang, "A generalized analysis technique of the stationary FPK equation in nonlinear systems under Gaussian white noise excitations," International Journal of Engineering Science, vol. 38, no. 12, pp. 1315-1330, 2000.

[5] R. Wang and K. Yasuda, "Exact stationary probability density for second order NonLinear systems under external white noise excitation," Journal of Sound and Vibration, vol. 205, no. 5, pp. 647-655, 1997.

[6] G. Q. Cai and Y. K. Lin, "Exact and approximate solutions for randomly excited MDOF non-linear systems," International Journal of Non-Linear Mechanics, vol. 31, no. 5, pp. 647-655, 1996.

[7] T. Caughey and F. Ma, "The exact solution of a class of nonlinear stochastic systems," International Journal of Nonlinear Mechanics, vol. 17, pp. 137-142, 1982.

[8] M. Dimentberg, "An exact solution to a certain nonlinear random vibration problem," International Journal of Nonlinear Mechanics, vol. 17, pp. 231-236, 1982.

[9] T. T. Soong, Random Differential Equations in Science and Engineering. New York: Academic Press, 1973.

[10] J. Johnson and R. Scott, "Extension of eigenfunction-expansion solution to a Fokker-Planck equation-II. second order systems," International Journal of Nonlinear Mechanics, vol. 15, pp. 41-56, 1980.

[11] J. D. Atkinson, "Eigenfunction expansions for randomly excited nonlinear systems," Journal of Sound and Vibrations, vol. 30, pp. 153-172, 1973.

[12] W. Mayfield, "A sequence solution to the Fokker-Planck equation," IEEE Transactions on Information Theory, vol. 19, no. 2, pp. 165 - 176, 1973.

[13] E. T. Jaynes, "Information Theory and Statistical Mechanics," Physical Review, vol. 106, no. 4, pp. 620-630, 1957.

[14] S. A. El-Wakil, E. M. Abulwafa, M. A. Abdou, and A. Elhanbaly, "Maximum-entropy approach with higher moments for solving FokkerPlanck equation," Physica A: Statistical Mechanics and its Applications, vol. 315, no. 3-4, pp. 480-492, 2002.

[15] S. A. El-Wakil, A. Elhanbaly, and M. A. Abdou, "Solution of FokkerPlanck equation by means of maximum entropy approach," Journal of Quantitative Spectroscopy and Radiative Transfer, vol. 69, no. 1, pp. 41-48, 2001.

[16] U. V. Wagner and W. V. Wedig, "On the calculation of stationary solutions of multi-dimensional Fokker-Planck equations by orthogonal functions," Nonlinear Dynamics, vol. 21, no. 3, pp. 286-306, 2000.

[17] G.-K. Er, "Exponential closure method for some randomly excited nonlinear systems," International Journal of Nonlinear Mechanics, vol. 35, no. 1 , pp. 69-78, 2000.

[18] _ - "A consistent method for the solution to reduced FPK equation in statistical mechanics," Physica A: Statistical and Theoretical Physics, vol. 262, no. 1-2, pp. 118-128, 1999.

[19] Q. Liu and H. G. Davies, "The non-stationary response probability density functions of non-linearly damped oscillators subjected to white noise excitations," Journal of Sound and Vibration, vol. 139, no. 3, pp. $425-435,1990$

[20] C. Soize, "Steady-state solution of Fokker-Planck equation in higher dimension," Probabilistic Engineering Mechanics, vol. 3, no. 4, pp. 196206, 1988.

[21] E. Hairer, S. P. Nørsett, and G. Wanner, Solving Ordinary Differential Equation I: Nonstiff Problems, $2^{\text {nd }}$ ed., ser. Springer Series in Computational Mathematics. Berlin, Germany: Springer Verlag, 2000.

[22] K. S. Shanmugan and A. M. Breipohl, Random Signals: Detection, Estimation and Data Analysis. New York, U.S.A.: John Wiley and Sons, 1988.

[23] R. F. Pawula, "Generalizations and extensions of the Fokker-PlanckKolmogorov equations," IEEE Transactions on Information Theory, vol. 13, no. 1, pp. 33-41, 1967. 